\title{
Reflexiones sobre el nivel de eficiencia energética de los edificios en Argentina y su relación con las emisiones de gases \\ de efecto invernadero
}

\author{
Reflections on the level of energy efficiency of buildings in \\ Argentina and its relation to emissions of greenhouse gases
}

Jorge Daniel Czajkowski *

Laboratorio de Arquitectura y Hábitat Sustentable. Facultad de Arquitectura y Urbanismo.

Universidad Nacional de La Plata / CONICET

layhs@fau.unlp.edu.ar 


\title{
Resumen
}

Uno de los aspectos de mayor relevancia en la actualidad es el cambio climático y la relación con las emisiones de gases de efecto invernadero. Diversos autores e instituciones tratan a nivel global y por países cual es la participación de los edificios, en cuanto a su construcción y mantenimiento, respecto al \% en las emisiones globales. En la mayoría de los casos a partir de datos globales que proveen empresas y gobiernos de cada país. En este caso los datos surgen del que oferta un servicio energético. No es usual tener la visión, desde la demanda, a partir de trabajo de campo y auditorías en edificios. En el trabajo se exponen resultados de auditorías tendientes a trazar un perfil del nivel de eficiencia energética y emisiones GEl de edificios y viviendas en Argentina. Se debate acerca de las acciones que se han emprendido para gestionar la mitigación del impacto del sector construcciones en las emisiones GEl y el cambio climático.

Palabras clave: Eficiencia energética; edificios, Gases de efecto invernadero; Uso racional de energía

\begin{abstract}
One of the most important aspects of today is climate change and for emissions of greenhouse gases. Many authors and institutions treat globally and countries for the participation of the buildings, in terms of construction and maintenance, as a percent in global emissions. In most cases from global data that provide businesses and governments in each country. In this case the data come from that offer an energy service. It is unusual to have the vision, since demand from fieldwork and audits in buildings. In the work results of audits aimed at profiling the level of energy efficiency and GHG emissions of buildings and houses in Argentina are exposed. There is debate about the actions that have been undertaken to manage construction impact mitigation GHG emissions sector and climate change.
\end{abstract}

Keywords: energy efficiency, buildings, energy use, climate change. 


\section{Introducción}

El trabajo fue desarrollado en el LAyHS - FAU UNLP y se enmarca dentro de una de las líneas de investigación principales que se desarrollan en el laboratorio, orientada hacia la evaluación y certificación de la eficiencia energética edilicia. Esto en el marco del los proyectos: UNLP 11/U141 - "Certificación de edificios sustentables para la adaptación y mitigación del cambio climático" y PIP CONICET "Protocolo de construcción de edificios e indicadores de sustentabilidad para la construcción del hábitat".

El trabajo busca hacer una reflexión sobre problemas actuales que afectan al país, como: la escasez de recursos, emisiones de gases de efecto invernadero y el calentamiento global. Estos están relacionados con la construcción de edificios y la ciudad, en grado significativo [IPCC, 2001]. Se ha encontrado que la industria de la construcción representa una de las más importantes consumidoras de materias primas y recursos no renovables e implica un sensible impacto ambiental, no sólo durante los procesos de extracción y elaboración de las materias primas, sino también durante la construcción de edificios, su utilización y aún después, cuando el edificio es demolido y reciclado [Edwards, 2008]. En el caso particular de Argentina los combustibles fósiles constituyen la principal fuente de energía empleada para la construcción y mantenimiento del hábitat construido. Implica el 96\% de la generación eléctrica mediante centrales de ciclo combinado y en climatización de edificios es intensivo el uso de gas natural [SEN, 2014].

EI WEC en su informe 2010 menciona que en la mayoría de las regiones del mundo se detectó una reducción de la intensidad energética en el período 1990 y 2008 que entienden, llevó a grandes ahorros de energía y por ende reducción de emisiones de CO2. Estiman en 3,6 Gtep en 2008 y 8 Gtep de emisiones de CO2. Además creen que muchas regiones del mundo tienen un potencial significativo para mayores reducciones en la intensidad energética. En el informe se menciona que a pesar de la implementación de políticas activas en el sector residencial, el ritmo del consumo eléctrico me- dio por hogar no solo no disminuyó sino que se muestran significativos aumentos. Principalmente en Latinoamérica el aumento de los ingresos del hogar favoreció el consumo de grandes electrodomésticos, como aparatos de refrigeración y lavado. Se sumaron nuevos dispositivos y aparatos, como el aire acondicionado. En muchos casos comercializando equipos menos eficientes. Además y gracias al impacto creciente de los países emergentes con bajos niveles de emisiones de CO2/cápita, las emisiones mundiales de CO2/cápita solo exponen un leve incremento de 4,2 ton CO2/capita (2008) respecto de 3,9 ton (1990) representando una variación del $+9 \%$.

El trabajo busca profundizar algunos aspectos relacionados principalmente en la eficiencia energética obtenible producto de la posible implementación de programas específicos de eficiencia energética y la estimación de emisiones de gases de efecto invernadero. Recordando que la Provincia de Buenos Aires es la única región que posee un instrumento de regulación de la eficiencia energética edilicia mediante la Ley 13059/2003 y su decreto reglamentario 1030/2010. La ciudad de Buenos Aires aprobó en 2012 la Ley 4458 pero sin reglamentar y la Ciudad de Rosario modificó su Código de Edificación. Solo esta última está implementando progresivamente una regulación energética en edificios nuevos o a remodelarse que en 2018 recién será para todo tamaño y función.

Desde el corriente año una nueva gestión nacional ha introducido varias innovaciones donde estos temas fueron incluidos en un Ministerio de Energía con rango de subsecretaría de Eficiencia Energética. Además se produjo un "sinceramiento" tarifario que generó gran intranquilidad social y es esperable que en el correr del año pasen a implementarse programas específicos.

\section{Metodología}

El trabajo parte de un método de manejo de datos estadísticos que surgen de Censos nacionales, 
junto a otros generados por Secretaría de Energía de la Nación, trabajos de autores varios y trabajos de investigación previos. Junto a un método simplificado de cuantificación de emisiones GEI a partir de la matríz energética nacional.

Además se utiliza información producto de trabajo de campo mediante auditorías en viviendas unifamiliares y edificios de departamentos. En la construcción de indicadores, índices y análisis edilicio se utilizaron los programas EnergoCAD y AuditCAD [Czajkowski, 1993, 2003]. Estos, permiten evaluar mes a mes el comportamiento térmico y energético de edificios, mediante el ingreso de los datos auditados al tiempo que determinan indicadores formales basados en Normas nacionales [IRAM 11603, 11605, 11604, 11659]. Se realizaron además evaluaciones de consumos de gas natural y electricidad para calefacción y refrigeración respectivamente.

Respecto a las campañas de monitoreos se realizaron en verano e invierno durante 7 días. Se utilizaron micro-adquiridores de datos HOBO U10-003 para medir la temperatura y humedad de los ambientes interiores. Para toma de datos del ambiente externo se usaron una Estación Meteorológica HOBO ProV2 y una estación meteorológica fija Davis "Vantage Pro2" (temperatura, humedad, radiación solar, vientos). La información se proceso con los programas dedicados "HOBOwarePro" y "BoxCarPro", junto a Excel.

Las características tecnológicas de un edificio junto a las condiciones climáticas del sitio de implantación determinan la cantidad de energía necesaria para acondicionar sus ambientes, sea en el período cálido como en el frío. Para obtener confort, y predecir y prevenir situaciones no deseables, es prudente conocer el posible comportamiento térmico del mismo y los requerimientos de energía [Filippín, 2005].

El sistema de calefacción usual en el país es el denominado "tiro balanceado" y resulta un equipo que consiste en una cámara de combustión estanca que intercambia aire y gases de combustión con el ambiente exterior, mientras que el calor es aportado interior de la vi- vienda. Estos calefactores tienen la ventaja de no consumir oxígeno ni aportan gases de la combustión al ambiente interior. La eficiencia térmica de calor transferido al ambiente se encuentra entre el $40 \%$ y el 60 \% [González y Juanicó, 2008]. Serán la referencia en la determinación de demandas potenciales en calefacción.

Se analizaron las demandas energéticas en el funcionamiento anual de los edificios y las emisiones de CO2 emitidas a la atmósfera. En dos escenarios: a. Para un sistema constructivo tradicional y b. Para una versión mejorada. Además, las pérdidas que se generan a través de las envolventes y los aportes de los factores que conforman carga térmica. Se realizó el análisis de las emisiones de $\mathrm{CO} 2$ proyectadas al ambiente que las viviendas generarían con ambas formas de construir. Para esto se utilizó un procedimiento simplificado desarrollado por el LAyHS [Marsilese\&Czajkowski, 2014] que usa el programa de cálculo creado por la US EnvironmentalProtection Agency (EPA) con la matriz energética de Argentina [González, 2010]. Debemos mencionar que los valores que da la calculadora de la EPA denominada Greenhouse Gas EquivalenciesCalculator, responden a la matriz energética de los EE.UU., donde gran parte de la generación eléctrica es por carbón. En Argentina, la fuente de generación eléctrica más difundida es la térmica y la hidroeléctrica, que en promedio es aproximadamente es un 50\% más limpia. Así debio adaptarse el ingreso de datos indicando un valor referido a: "litros de gasolina consumidos", "kWh de electricidad", "termias de gas natural", etc., luego se obtiene el resultado en dióxido de carbono o CO2 equivalente. En nuestro caso, los resultados fueron:

$$
E C O 2 O 1=0.27 \mathrm{TnCO} 2 / \mathrm{m} 2 \text { (Eq 1) }
$$$$
\text { ECO2O2 }=0.31 \mathrm{TnCO} 2 / \mathrm{m} 2(E q \text { 2) }
$$

\section{Resultados:}

\section{Sobre los escenarios tecnológico constructivos:}

En el caso de la vivienda tipo de construcción tradicional, las pérdidas a través de la envol- 
vente se distribuyeron de la siguiente manera: Cubierta: 39\% (762W/K); Muros: 14\% (270W/K); Ventanas: 18\% (342W/K); Puertas: 3\% (54W/K); Pisos: $4 \%(86 \mathrm{~W} / \mathrm{K})$ y Renovaciones de aire (2RA): 22\% (426W/K). En el caso mejorado, las pérdidas se distribuyeron de esta manera: Cubierta: $\quad 44,1 \%(381 \mathrm{~W} / \mathrm{K})$; Muros: 15,6\% $\left(195 \mathrm{~W} /{ }^{\circ} \mathrm{C}\right)$; Ventanas : 15,2\% (132W/K); Puertas : 3,9\% (34W/K) y Pisos : 4,7\% (41W/K) y Renovaciones de aire (2RA): 16,4\% (192W/K).

Para el escenario mejorado se usó el promedio entre nivel A y B de la IRAM 11605 en muros y cubiertas, en ventanas doble vidriado hermético y marcos de prestaciones medias y en pisos Nivel B. Es una hipótesis de aplicar la Ley 13059 ligeramente mejorada a todo el país a una vivienda unifamiliar media ponderada. Implica $0,10 \mathrm{~m}$ de aislamiento en cubiertas ( $\mathrm{R}=2.85 \mathrm{~m} 2 \mathrm{~K} / \mathrm{W}), 0,05 \mathrm{~m}$ de aislamiento térmico en muros $(\mathrm{R}=1.43 \mathrm{~m} 2 \mathrm{~K} / \mathrm{W}) \mathrm{y}$ DVH en vidriados $(\mathrm{R}=0.54 \mathrm{~m} 2 \mathrm{~K} / \mathrm{W})$.

La Figura 1 muestra la variación de pérdidas de calor unitarias en relación a las partes de envolvente de una vivienda tipo media ponderada de 53,2 m2 para un caso construido de forma standard y otra mejorada cumpliendo normas nacionales.

Figura 1: Distribución de pérdidas según balance térmico para vivienda tipo media ponderada caso standard y mejorado.

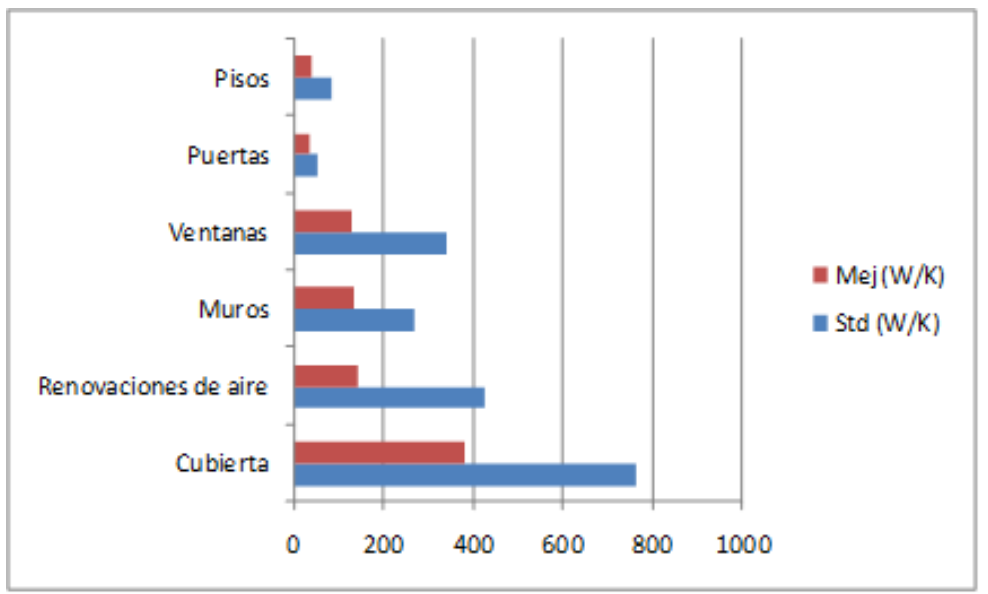

Fuente: Elaboración propia.

Tabla 1: Demanda potencial de energía en calefacción para zonas bioclimáticas II a VI

\begin{tabular}{|l|l|l|l|l|}
\hline $\begin{array}{l}\text { Grados día } \\
\left.\text { (base } 18^{\circ}\right)\end{array}$ & $\begin{array}{l}\text { Vivienda Std } \\
(\mathrm{kW} / \text { año) }\end{array}$ & $\begin{array}{l}\text { Vivienda Mej } \\
\text { (kW/año) }\end{array}$ & $\begin{array}{l}\text { Vivienda Std } \\
(\mathrm{kW} / \mathrm{m} 2 . a n ̃ o)\end{array}$ & $\begin{array}{l}\text { Vivienda Mej } \\
(\mathrm{kW} / \mathrm{m} 2 . \text { año) }\end{array}$ \\
\hline 500 & 970 & 432 & 18,2 & 8,1 \\
\hline 1000 & 1940 & 864 & 36,5 & 16,2 \\
\hline 1500 & 2910 & 1296 & 54,7 & 24,4 \\
\hline 2000 & 3880 & 1728 & 72,9 & 32,5 \\
\hline 2500 & 4850 & 2160 & 91,2 & 40,6 \\
\hline 3000 & 5820 & 2592 & 109,4 & 48,7 \\
\hline 3500 & 6790 & 3024 & 127,6 & 56,8 \\
\hline 4000 & 7760 & 3456 & 145,9 & 65,0 \\
\hline 4500 & 8730 & 3888 & 164,1 & 73,1 \\
\hline 5000 & 9700 & 4320 & 182,3 & 81,2 \\
\hline 5500 & 10670 & 4752 & 200,6 & 89,3 \\
\hline
\end{tabular}

Fuente: Elaboración propia. 
Figura 2: Comparación de cargas térmicas en kW/año (y) para vivienda en función de los grados día de calefacción `D/año (x).

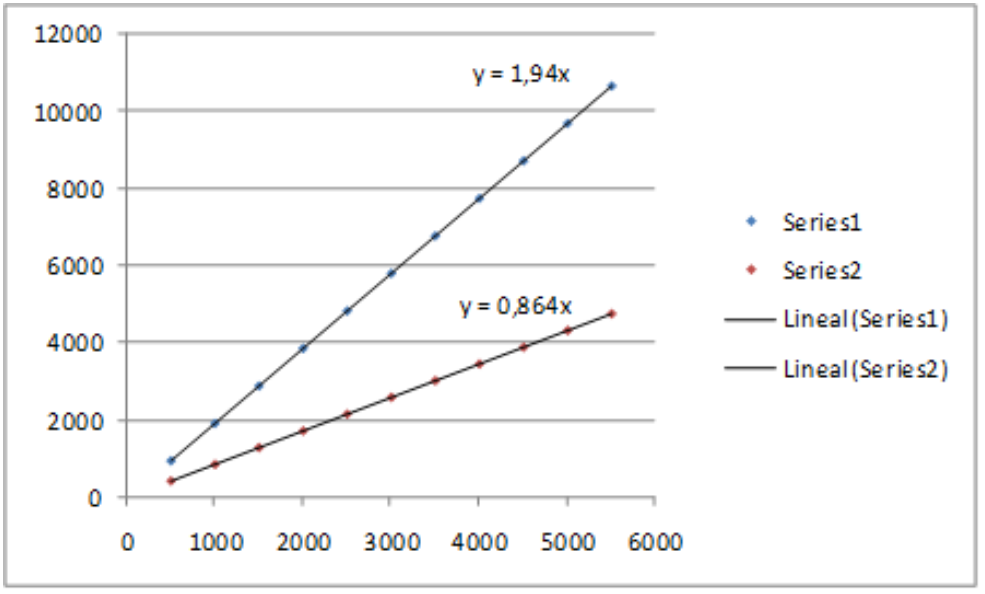

Fuente: Elaboración Propia.

Figura 3: Comparación de cargas térmicas en kW/m2.año (y) para vivienda en función de los grados día de calefacción D/año (x)

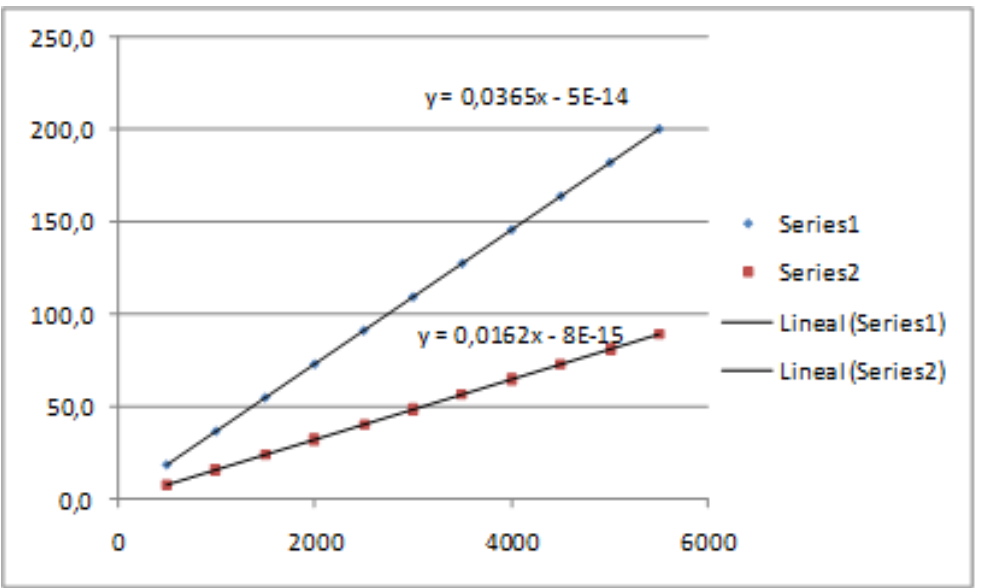

Fuente: Elaboración Propia.

Se determina la demanda potencial de energía en calefacción para las zonas climáticas Argentinas donde la Norma IRAM 11604 considera que se requiere contar con un sistema de calefacción, siendo las zonas II a VI. Estas son templado cálido a muy frío indicado en Tabla 1. En Figura 2 se obtienen las rectas de correlación lineal para los casos de vivienda standard y vivienda mejorada. Donde resulta que puede estimarse la demanda de energía en calefacción a partir de cada escenario. Así:

Qstd (kWh/año) = 1,94 x Grados Día de Calefacción (base $18^{\circ} \mathrm{C}$ ) (Eq 3)
Qmej (kWh/año) =0,864 x Grados Día de Calefacción (base $18^{\circ} \mathrm{C}$ ) (Eq 4)

La Figura 3 muestra una comparación de correlaciones lineales entre los grados día de calefacción y la demanda potencial de energía en calefacción en kWh/m2.año para los casos standard y mejorado.

Análisis para casos tipo casa y departamento:

La tabla 2 muestra una síntesis de valores medios de coeficiente Gmcal (W/m3K) [IRAM 
Tabla 2: Tipos de vivienda y sus características formales y térmicas

\begin{tabular}{lcccccc}
\hline & $\mathrm{Gm}_{\text {cal }}$ & $\mathrm{Vm}$ & $\mathrm{Sm}$ & Peso & Tcal & FCV \\
\hline Casa compacta & 2,98 & 180 & 67 & 60 & 12 & 0,45 \\
Departamento & 2,5 & 100 & 37 & 30 & 12 & 0,7 \\
Casa grande & 2,15 & 350 & 130 & 10 & 24 & 0,7 \\
\hline
\end{tabular}

Fuente: Elaboración propia.

11604], donde se representan para cada tipo de vivienda, el volumen calefaccionado medio Vm (m3), superficie calefaccionada media Sm (m2), peso porcentual de participación en el tejido urbano en ciudades medias a grandes, el tiempo medio de calefacción según encuestas Tcal (hs/día) y el factor de \% de calefacción medio de las viviendas. Se agrupan los casos auditados en tres segmentos a los que se denominan como casa compacta, departamento y casa grande.

De las auditorías surge que los tres tipos de viviendas tendrán un consumo específico de gas natural de $1,2 \mathrm{~m} 3 /{ }^{\circ} \mathrm{C}$. día para el tipo Casa compacta, $0,56 \mathrm{~m} 3 /{ }^{\circ} \mathrm{C}$. día para el tipo Departamento y $2,36 \mathrm{~m} 3 /{ }^{\circ} \mathrm{C}$.día para el tipo Casa grande. Con los pesos de la tabla 1 se ha determinado el consumo específico de gas natural en calefacción ponderado CCp según la siguiente expresión:

$$
\begin{gathered}
\text { CC ponderado }=0,54 \times 0,6+0,56 \times 0,3+2,36 \times \\
0,1=0,73 \mathrm{m3} /{ }^{\circ} \mathrm{C} \text { día }(E C .5)
\end{gathered}
$$

Del análisis surge que el valor de $0,73 \mathrm{~m} 3 /{ }^{\circ} \mathrm{C}$. día, resulta de las características de las viviendas y de realizar un cálculo a partir de valores medios ponderados de la muestra. Se ha encontrado que sea en casas compactas o en departamentos hay un porcentaje significativo que no climatiza la vivienda o lo hace mínimamente. Principalmente en las zonas del país con bajos a nulos subsidios en mayoritariamente en las Zonas bioclimáticas II a IV. En las determinaciones no se ha considerando el efecto del aporte solar medio local sino que se partió de calcular en un 12\% para un 30\% de días soleados en los meses de invierno. Esto haría reducir un poco este valor. Esto nos lleva luego a poder comparar casos si los tuviéra- mos a temperatura de termostato respecto de casos auditados que no alcanzan un confort térmico interior mínimo.

Al comparar el indicador obtenido en este trabajo con el que elabora el Ente Nacional Regulador del Gas vemos diferencias ya que ENARGAS lo estima en $0,56 \mathrm{~m} 3 /^{\circ} \mathrm{C}$.día. Esto nos muestra varias cosas: a. por una parte el valor medio de ENARGAS es un 30\% inferior al obtenido por este trabajo mediante auditorías e implica que se calefacciona menos de lo que se ha encuestado; b. otra posibilidad es que la muestra auditada tiene una tendencia hacia sectores económicos medios a medios altos. Lo cual implica que es significativo el impacto de los sectores de bajos recursos que habitan viviendas de similares características tanto en dimensiones como en sistemas constructivos.

La diferencia principal es que las estadísticas oficiales dicen que se consume menos y esto solo se puede lograr por menor tiempo de calefacción, menor superficie calefaccionada y por ende temperaturas medias interiores muy por debajo de un confort a $18^{\circ} \mathrm{C}$. Entre otras posibles hipótesis.

Ya se ha mencionado que en el país existen leyes, decretos reglamentarios y hasta códigos de edificación que exigen el cumplimiento de las Normas que tenderían a regular la calidad térmica edilicia. También se mencionó que en realidad no se cumplen. No lo hace el sector público ni el sector privado.

Sobre lo planteado se presentan tres escenarios distintos para mostrar la demanda media anual por unidad habitacional en el Área Metropolitana de Buenos Aires con acceso a gas natural por red. Esto con el fin de poder 
Figura 4: Comparación de la demanda de energía específica en calefacción (IDEcal), en viviendas tipo medias en tres escenarios nacionales de consumo y casos de otros países.

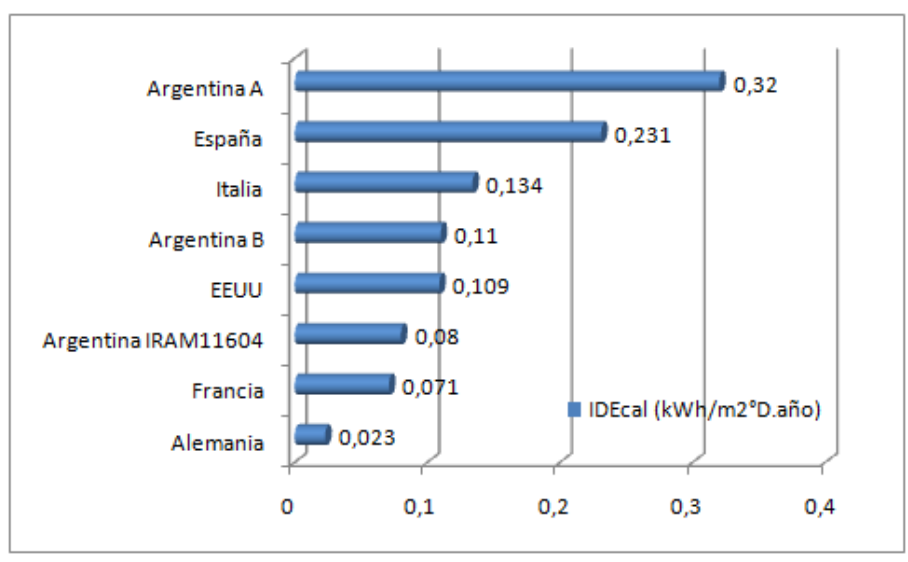

Fuente: Elaboración Propia.

Figura 5:Comparación de las emisiones de CO2 en TnCO2/m2.año (y) para viviendas en función de los grados día de calefacción º/año (x). Fuente: Elab Propia.

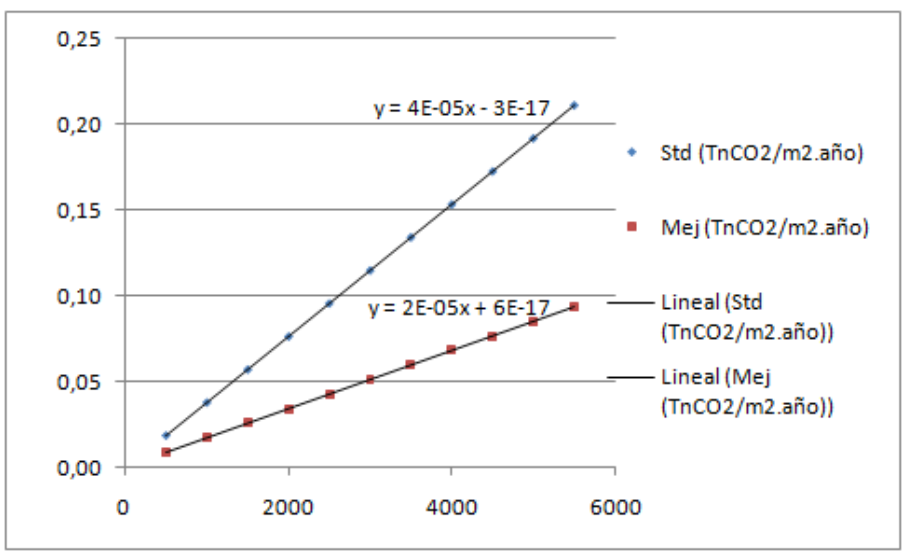

Fuente: Elaboración propia.

comparar una vivienda nacional respecto a otras a nivel internacional.

En la Figura 4 puede verse que la vivienda tipo media que representa a las más de 3,62 millones de viviendas del AMBA es un 28\% (0,32 $\mathrm{kWh} / \mathrm{m} 2^{\circ} \mathrm{D}$ año) más ineficiente que una vivienda media española $\left(0,23 \mathrm{kWh} / \mathrm{m} 2^{\circ} \mathrm{D}\right.$ año) y un $93 \%$ más ineficiente que una vivienda alemana $\left(0,023 \mathrm{kWh} / \mathrm{m} 2^{\circ} \mathrm{D}\right.$ año). Siempre tomando la vivienda media construida según los Códigos de Edificación vigentes en la región.

Dado que no se tiene conocimiento de exigencias para regular la calidad térmica de la construcción en el país en viviendas que no correspondan a planes oficiales, podemos asumir a Argentina a como un valor medio nacional.

\section{Acerca de las emisiones GEI de $\mathrm{CO} 2$ en viviendas}

En cuanto a emisiones GEl y más en particular en lo concerniente a $\mathrm{CO} 2$ podemos utilizar los índices desarrollados por Marcilese expresados en las ecuaciones 1 y 2 para los casos de viviendas tipo standard o mejorada. Estos coeficientes contemplan las emisiones debidas al funcionamiento de la misma en calefacción junto a las emisiones que devienen del ciclo de vida de los materiales con los cuales se construye una vivienda. Otra alternativa es utilizar un indicador energético-ambiental como el desarrollado por Discoli para la matriz energética nacional (Discoli, 1998). En este caso estima que:

$$
\begin{gathered}
\text { CO2 [kg/año] = Energía eléctrica [kWh] } \\
/ 11600 \times 3050 \times 4[E c .6]
\end{gathered}
$$


Mediante los valores de demanda de energía calculados en Tabla 1 y mediante la siguiente expresión podemos conocer cual serán las emisiones de CO2/m2.año en nuestra vivienda tipo en sus versiones convencional y mejorada.

\section{EV-CO2 [tn/ $/ \mathrm{m} 2$.año] = Energía eléctrica [kWh/año] $\times 3050 \times 4 / 11600000 / 53,2$ [m2] [EC. 6]}

La figura 5 muestra la relación entre grados día de calefacción base $18^{\circ} \mathrm{C}$ y las emisiones potenciales de $\mathrm{CO} 2$ para la vivienda tipo analizada en el trabajo en su condición standard que en Figura 4 denomináramos Argentina A respecto de la misma mejorada. Las expresiones que surgen permiten determinar para cualquier ciudad o zona bioambiental del país las emisiones de CO2 por metro cuadrado construido.

\section{Conclusión}

En el trabajo se ha buscado determinar nuevos indicadores que permitan conocer las demandas potenciales de energía en calefacción y las emisiones que derivan de estas. Es posible continuar a fin de conocer para cada ciudad o provincia con el método elaborado las emisiones GEl aunque excede el artículo. Debatimos sobre los indicadores energéticos usualmente generados desde los organismos nacionales que solamente apuntan al mercado de la energía sin mayor profundidad. Es necesario que el país pueda contar en tiempo corto (mes, año) valores de oferta y demanda de energía como de emisiones GEl equivalentes. Para hacer eficiencia energética se requiere información y ésta prácticamente no existe. Solo pueden hacerse estimaciones debido a la férrea oposición de las empresas prestadoras de servicios energéticos de dar información. También debieron realizarse estimaciones de cantidad de viviendas al presente ya que en el Censo Nacional de 2001 se alcanzó un detalle que lamentablemente en 2010 solo sirvió para saber cuántos somos y no a nivel de ciudades y mucho menos por casas o departamentos. Son tantos los inconvenientes en un país sin estadísticas o con estadísticas de baja calidad que solo queda tratar de obtener información estimada o global. Se ha encontrado que en todos los casos las estadísticas son con una visión desde la oferta de energía. En ningún caso son utilizados indicadores desde la demanda, o que permitan inferir el grado de utilización de la energía en edificios en relación a la satisfacción de un cierto grado de confort. Tampoco es posible cuantificar si existe un yacimiento potencial de ahorro y uso racional de la energía y si este ahorro es factible. Los indicadores propuestos en este trabajo y en otros citados buscan mejorar la calidad de información centrado en la demanda. Son indicadores construidos a partir del análisis de casos construidos y funcionando que han sido auditados. Contar con instrumentos de regulación de la eficiencia energética edilicia sean normas, leyes, reglamentos o códigos de edificación no es suficiente si la política del estado sea nacional, provincial o municipal es hacia la desregulación o a la regulación por vía del incremento tarifario. Esta falta de política energética para regular la demanda tiende al quebranto energético y económico, perpetuación de las crisis energéticas, falta de innovación en la construcción, aumento de las emisiones GEl, entre otros; que son una muestra de la falta de desarrollo de un país. 
Bibliografía

Czajkowski, Jorge Daniel; Gómez, Analía Fernanda. (2009). Herramientas para arquitectos: Arquitectura Sustentable. Ciudad Autónoma de Buenos Aires: Arte Gráfico Editorial Argentino. 2009. Pag.144. ISBN 978-987-07-0603-8

Czajkowski, J. (1993). Desarrollo del programa AuditCAD para el análisis de edificios a partir de auditorías ambientales. Revista Avances en energías renovables y ambientales (3). ISSN 0329-5184.

Czajkowski, Corredera, Saposnik. (2003). Análisis de la relación entre demanda de gas natural en calefacción según "EnergoCAD" y consumos reales en viviendas unifamiliares del Gran La Plata. Revista Avances en energíasrenovables y ambiente (7). ISSN 0329-5184.

Czajkowski, J. (2015) Energy Efficiency Indicators for Buildings in Argentina. In book: The Sustainable Renovation of Buildings and Neighbourhoods. ISBN: 978-1-68108-065-9.

Discoli, Carlos (1998). El diagnóstico de la gestión productiva energético-ambiental de las redes territoriales del sector salud. Edit UNLP, La Plata.

Edwards, Brian. (2008). Guía básica de la sostenibilidad. Gustavo Gili, SL. Barcelona. ISBN 8425222087.

Filippin, C. (2005) Thermal Response of Solar and Convencional School Building to Design-and- Human-Driven Factors. RenewableEnergy 30, 353-376.

González, Alejandro. (2010) Comparación de energías y gases de efecto invernadero en calentamiento de agua para cocción de alimentos con electricidad y gas natural. Avances en Energías Renovables y Medio Ambiente.

González, Alejandro; Juanicó, L y Gortari, S. (2008) High-EfficiencyPrototypes of Commercial Gas HeatersExtensivelyUsed in Argentina. ScienceDirect. International Journal of HydrogenEnergy, 33.

INDEC (2015). Estimaciones y proyecciones de población 2010-2040. Total del país.

International Energy Agency. (2015) Indicadores de Eficiencia Energética. Bases esenciales para el establecimiento de políticas. Recuperado de : https://www.iea.org/publications/.../EnergyEfficiencyVespagnol_epdf.pdf

IPCC. (2001). Tercer informe de evaluación del Intergovernmental Panel onClimateChange: Cambio Climático. Ginebra, Suiza. ISBN 0521807700.

Marsilese, Mariela y Czajkowski, Jorge (2014). Análisis simplificado de la eficiencia energética de una escuela rural en la pampa - Argentina - a lo largo de su ciclo de vida. Revista Hábitat Sustentable 3, (1), , p. 3-14 ISSN: $0719-0700$

Norma IRAM 11601:2002. Aislamiento térmico de edificios, métodos de cálculo. Propiedades térmicas de los componentes y elementos de construcción en régimen estacionario. IRAM. Buenos Aires.

Norma IRAM 11603:1996. Acondicionamiento térmico de edificios. Clasificación bioambiental de la República Argentina. IRAM. Buenos Aires.

Norma IRAM 11605:1996. Acondicionamiento térmico de edificios, condiciones de habitabilidad en edificios. Valores máximos de transmitancia térmica en cerramientos opacos. IRAM. Buenos Aires.

Norma IRAM 11900:2010. Etiqueta de eficiencia energética de calefacción para edificios. Clasificación según la transmitancia térmica de la envolvente. IRAM. Buenos Aires.

SEN, Secretaría de Energía de la Nación. (2014). Balance Energético Nacional 2013. Ministerio de Economía de la Nación. Buenos Aires. Recuperado: http://www.energia.gov.ar/... /balances_2014/Ben13. $\mathrm{xls}$

WEC - Consejo Mundial de Energía. (2010) Eficiencia Energética: Una Receta para el Éxito - Resumen Ejecutivo. ISBN: 9780946121007 